\title{
Cycling Behavior and Memory Formation
}

\author{
Jason R. Gerstner, ${ }^{1}$ Lisa C. Lyons, ${ }^{2}$ Kenneth P. Wright Jr, ${ }^{3}$ Dawn H. Loh, ${ }^{4}$ Oliver Rawashdeh, ${ }^{5}$ Kristin L. Eckel-Mahan, ${ }^{6}$ \\ and Gregg W. Roman ${ }^{7}$ \\ ${ }^{1}$ Department of Genetics, University of Wisconsin-Madison, Madison, Wisconsin 53706, ${ }^{2}$ Department of Biological Science, Florida State University, \\ Tallahassee, Florida 32306, ${ }^{3}$ Department of Integrative Physiology, University of Colorado, Boulder, Colorado 80309, ${ }^{4}$ Department of Psychiatry and \\ Biobehavioral Sciences, University of California, Los Angeles, Los Angeles, California 90024, ${ }^{5}$ Department of Pharmacology and Toxicology, University at \\ Buffalo, State University of New York (SUNY), Buffalo, New York 14214, ${ }^{6}$ Department of Pharmacology, University of California, Irvine, Irvine, California \\ 92697, and 7Department of Biology and Biochemistry, University of Houston, Houston, Texas 77204
}

Circadian research has spent considerable effort in the determining clock output pathways, including identifying both physiological and behavioral processes that demonstrate significant time-of-day variation. Memory formation and consolidation represent notable processes shaped by endogenous circadian oscillators. To date, very few studies on memory mechanisms have considered potential confounding effects of time-of-day and the organism's innate activity cycles (e.g., nocturnal, diurnal, or crepuscular). The following studies highlight recent work describing this interactive role of circadian rhythms and memory formation, and were presented at a minisymposium at the 2009 annual meeting of the Society for Neuroscience. The studies illustrate these time-of-day observations in a variety of behavioral paradigms and model organisms, including olfactory avoidance conditioning in Drosophila, long-term sensitization in Aplysia, active-avoidance conditioning in Zebrafish, and classical fear conditioning in rodents, suggesting that the circadian influence on memory behavior is highly conserved across species. Evidence also exists for a conserved mechanistic relationship between specific cycling molecules and memory formation, and the extent to which proper circadian cycling of these molecules is necessary for optimal cognitive performance. Studies describe the involvement of the core clock gene period, as well as vasoactive intestinal peptide, melatonin, and the cAMP/MAPK (cAMP/mitogen-activated protein kinase) cascade. Finally, studies in humans describe evidence for alterations in cognitive performance based on an interaction between sleep-wake homeostasis and the internal circadian clock. Conservation of a functional relationship between circadian rhythms with learning and memory formation across species provides a critical framework for future analysis of molecular mechanisms underlying complex behavior.

\section{Introduction}

In most systems examined in detail, the core molecular circadian oscillator exists as an interlocked transcriptional auto-regulatory feedback loop. This oscillator receives inputs from zeitgebers, such as light, and the output provides time-of-day information for many physiological and behavioral processes. The detailed understanding of this complicated molecular clock arose from a comparative approach. Genetic screens beginning with the observations of a daily rhythm in the emergence of Drosophila from their pupal case (eclosion rhythm) (Konopka and Benzer, 1971) led eventually to the isolation and characterization of the first circadian gene period (Bargiello et al., 1984; Reddy et al., 1984). Additional genes identified by mutations from Drosophila, Neurospora, Syrian hamster and mouse helped to fill out the components of the core oscillator. Many components of the vertebrate oscillator were first identified by homology to Drosophila genes, and allowed for the molecular dissection of their operations. The phylogenetic conservation of the core components of the molecular oscillator allowed investigations in simpler model

Received July 13, 2009; revised Aug. 13, 2009; accepted Aug. 24, 2009.

Correspondence should be addressed to Jason R. Gerstner, 3462 Genetics/Biotechnology, University of Wisconsin-Madison, 425 Henry Mall, Madison, Wisconsin 53706. E-mail: jrgerstn@wisc.edu. D01:10.1523/JNEUROSCI.3353-09.2009

Copyright $\odot 2009$ Society for Neuroscience $\quad 0270-6474 / 09 / 2912824-07 \$ 15.00 / 0$ systems to inform mechanistic studies in more complex mammalian systems (Panda et al., 2002; Gallego and Virshup, 2007).

Memory formation and consolidation are often classified by a relationship with time. Multiple species exhibit temporal windows for various forms of memory. These include short-term memory (STM), which can last from seconds to hours, and longterm memory (LTM), which can last from hours to days, months, or even years (McGaugh, 2000). A putative mechanism for the emergence of LTM out of STM was proposed by both Hebb (1949) and Gerard (1949), dubbed the "dual-trace theory" of memory, where reverberating neural activity of STM is thought to stabilize to form longer-lasting LTM. Cellular and molecular evidence also suggest that these two forms of memory are somewhat distinct, in that inhibitors of protein synthesis are able to disrupt LTM without affecting STM (McGaugh, 2000; CostaMattioli et al., 2009). Exactly if or how these cellular processes and molecules might play an integrated role in stabilizing STM to produce longer-lasting LTM is not well known. Circadian cycling is one method that has recently been proposed, through the time-of-day oscillations, or "reverberations," of specific molecules after learning for proper memory consolidation (Roth and Sweatt, 2008).

Since there is a core set of genes in multiple species that are known to regulate circadian rhythms, are these circadian genes also able to regulate the formation of memory? Given that the 
Table 1. Phylogenetically organized list of species with observed time-of-day regulated memory, and their molecular correlates

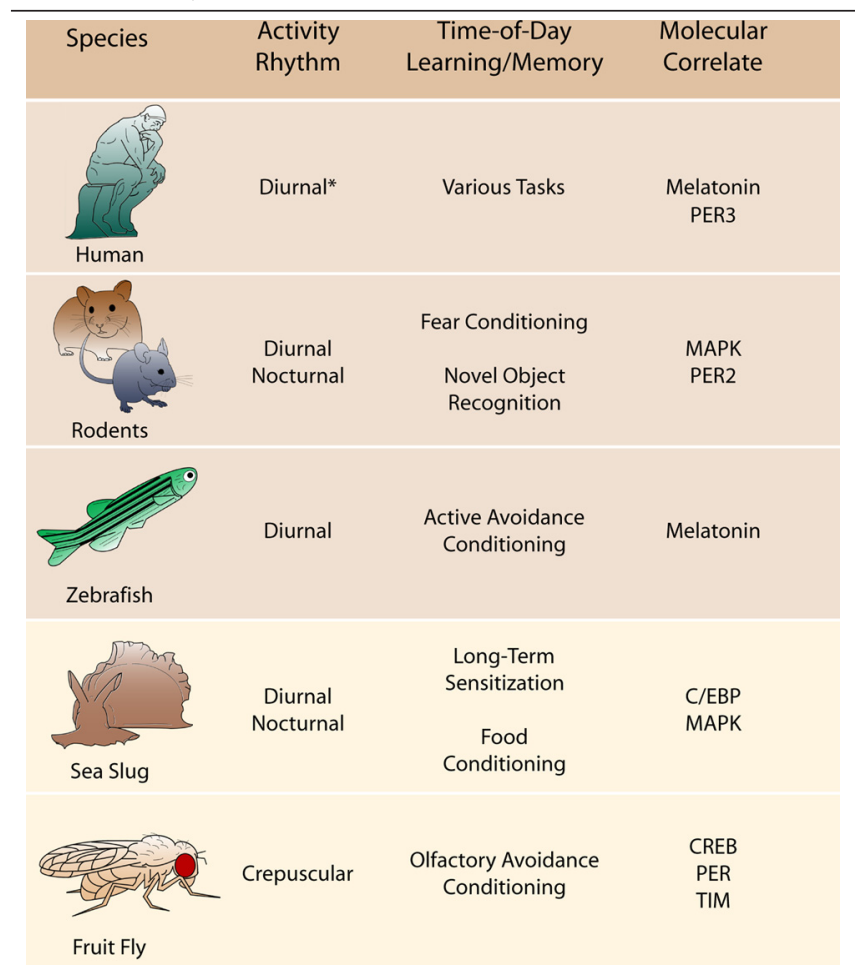

Species discussed within this mini-symposium review share common molecular correlates in their time-of-day regulation of memory, despite differences in their respective activity rhythm. PER3, Period 3; PER2, period 2; C/EBP, CCAAT-enhancer-binding protein; PER, period; TIM, timeless. *Activity rhythm is varied based on an increasingly $24 \mathrm{~h}$ society.

time-of-day regulation of memory has been observed in a variety of invertebrate and vertebrate species (Table 1), it should not be surprising that genes of the core oscillator, such as period, have been shown previously to regulate memory formation (Sakai et al., 2004), in addition to other complex behavior, such as drug conditioning (Andretic et al., 1999; Abarca et al., 2002). A considerable remaining question is how the oscillator modulates the formation and consolidation of different memories. The purpose of this review is to collate recent developments in the study of the circadian regulation of memory formation. The contributions summarized below offer examples of the time-of-day influence on memory behavior. We describe these circadian effects on memory in various alternative model organisms, and span the various chronobiological categories of diurnal, nocturnal, and crepuscular activity rhythms (Table 1). We also provide some evidence for phylogenetic conservation in specific molecular and genetic mechanisms that contribute to these observations. By presenting these data in this review, we hope to bring attention to the importance of considering the influence of time-of-day on memory formation (and other complex behavior) to the broader neuroscience field.

\section{Short-term olfactory memory in Drosophila is modulated by an endogenous circadian clock}

Circadian oscillators extensively modulate cognitive processes in a phase-specific manner; these effects can be robust even after accounting for fatigue and sleep deprivation (Monk and Folkard, 1978; Wright et al., 2006). The circadian modulation of longterm memories generated in different learning paradigms has been found in both vertebrates and invertebrates (Rudy and
Pugh, 1998; Valentinuzzi et al., 2004; Fernandez et al., 2003; Lyons et al., 2005; Rawashdeh et al., 2007). A role for the endogenous circadian clock in modulating short-term memory formation has been appreciably more difficult to demonstrate.

The negatively reinforced olfactory learning paradigm of Drosophila melanogaster was used to investigate the role of circadian modulation of short term memory formation (Lyons and Roman, 2009). This olfactory learning paradigm is a form of classical conditioning that involves the association of an electric shock (US), paired with a particular odor, the CS+ (for review, see Roman and Davis, 2001). This pairing is subsequently followed by the presentation of an unpaired second odorant (CS-); learning is measured in a T-maze as the avoidance of the $\mathrm{CS}+$ relative to the CS - . The number of US/CS + pairings determines the strength of memory formed; a single US/CS + pairing produces modest levels of learning, whereas 12 pairings produces asymptotic learning (Tully and Quinn, 1985; Beck et al., 2000).

A rhythm in short-term associative memory was found during both light-dark cycles (LD) and constant darkness (DD) using the negatively reinforced olfactory learning paradigm (Lyons and Roman, 2009). The performance of wild type flies in this assay displayed a broad peak during the early subjective night. Mutations in both timeless and period genes that disrupt the central circadian oscillator also disrupt the rhythm in short-term associative memory. The rhythm in short-term memory is also disrupted in constant light conditions (LL); constant light disrupts the central circadian oscillator of Drosophila (Konopka et al., 1989; Marrus et al., 1996). Hence, the endogenous circadian clock is necessary for the rhythm in short-term associative memory. The amplitude of the rhythm in short-term memory was approximately twofold greater when the flies received only a single pairing of CS + and US compared with the rhythm found with asymptotic learning (12 pairings of CS + and US), suggesting that the circadian clock may be modulating the rate of acquisition. It is unlikely however, that a rhythm in perception of the stimuli used in these assays underlies the short-term memory rhythm. The naive olfactory and shock avoidance behaviors are not regulated by time-of-day under the conditions used in these assays. Moreover, a mutation in cryptochrome that disrupts the antennal circadian oscillators does not disrupt the circadian rhythms in short-term memory (Krishnan et al., 2001; Lyons and Roman, 2009). Interestingly, a rhythm with similar phase has also been found in the nocturnal cockroach, Leucophaea maderae, for 30 min positively reinforced olfactory memory (Decker et al., 2007). Thus, the data strongly argue for a general role of the circadian oscillator in modulating short-term associative memories.

\section{Circadian modulation of memory in Aplysia}

Research in gastropod mollusks has provided key insights into the neural and molecular mechanisms underlying the formation of memory. Behavioral studies in these comparatively simple invertebrates offer the means for understanding the modulation of memory and the opportunity for identification of manipulated processes. The wide array of available learning paradigms has made Aplysia an outstanding model for analyzing circadian modulation of memory.

The circadian clock broadly impacts memory formation in Aplysia for nonassociative and associative learning. Circadian modulation of long-term sensitization of the tail-siphon withdrawal reflex results in animals demonstrating significant memory when trained during the (subjective) day and little long-term memory when trained at night (Fernandez et al., 2003). Importantly, the circadian clock modulates formation of memory, 
rather than perception of sensory stimuli or recall. Similarly, the circadian clock strongly modulates long-term associative memory for an operant paradigm, learning that food is inedible (LFI). Diurnal Aplysia californica exhibit robust long-term memory when trained during the day, and virtually no long-term memory at night (Lyons et al., 2005). Conversely, nocturnal Aplysia fasciata demonstrate long-term memory only when trained during the night. Thus, the circadian clock modulates nonassociative and operant learning with comparable phase restrictions, suggesting that temporal coordination of multiple behaviors including activity, feeding, and long-term memory results in significant adaptive advantages.

Short-term memory appears outside of the purview of circadian modulation in Aplysia. This lack of regulation could be due to the nature of the learning, i.e., behaviors for which repression of short-term memory could have disastrous consequences. However, a more likely possibility arises when the mechanisms underlying short and long-term memory are considered. The formation of long-term memory involves persistent kinase activation, protein synthesis, and new gene expression. This suggests that plasticity exists within these critical processes that may be subject to modulation. Lyons et al. (2006a) sought to pinpoint discrete molecular steps through which the circadian clock targeted memory. Importantly, they identified circadian restrictions on training-induced activation of cAMP/mitogen-activated protein kinase (MAPK) signaling and new gene expression resulting in the suppression of long-term memory at night. Intriguingly, the circadian clock appears to modulate memory at multiple levels as ameliorating the suppression of memory required pharmacological facilitation of both MAPK signaling and transcription (Lyons et al., 2006a). These experiments present the possibility that targeting of specific pathways may be effective in alleviating adverse effects on learning due to phase-specific circadian suppression of memory.

Thus, a picture emerges in which circadian modulation of long-term memory appears coordinated with the animal's activity period through the targeting of conserved processes. This suggests that intermediate-term memory, a distinct form of memory also dependent upon persistent kinase activity and protein synthesis, may be regulated by the circadian clock. Indeed, recent studies have demonstrated circadian modulation of intermediate sensitization (Lyons et al., 2008) and new data presented at the 2009 annual meeting of the Society for Neuroscience suggest strong circadian modulation of intermediate LFI memory (Green et al., 2009). While phase similarities exist between intermediate and long-term memory, phase differences likely reflect mechanistic divergence in the formation of intermediate and long-term LFI memory. Hence, these studies also suggest that the circadian clock modulates memory formation at multiple steps.

\section{Circadian modulation of long-term memory by melatonin in the diurnal zebrafish, Danio rerio}

Locomotor activity (Cahill et al., 1998; Hurd et al., 1998), feeding behavior (Stokkan et al., 2001), core body temperature (Castillo et al., 2005) and memory processes (Chaudhury and Colwell, 2002; Fernandez et al., 2003; Lyons et al., 2005) are all examples of downstream outputs modulated by an animals' circadian system. Evidence for a circadian influence on learning and memory was initially demonstrated indirectly by disrupting the circadian organization and testing for the ability to learn or recall an acquired task (Tapp and Holloway, 1981; Fekete et al., 1985; Antoniadis et al., 2000; Devan et al., 2001).
Many processes that influence memory, such as protein and neurotransmitter synthesis, synaptic activity, and hormone secretion, show circadian oscillations (Scheving et al., 1968; Barnes et al., 1977; Johnson et al., 1972). Thus, memory processes could be temporally gated downstream of some circadian regulated biochemical process. Rawashdeh et al. (2007) investigated whether, in the diurnal zebrafish (Danio rerio), different memory processes such as acquisition, memory formation and retrieval were specifically restricted or facilitated during certain times of day. Using a modified active avoidance conditioning (AAC) training paradigm, a form of operant conditioning, in which animals learn to associate an environment associated with dim red light (conditional stimulus) being safe or a dark environment associated with mild electric shocks (unconditional stimulus) as being unsafe, they were able to demonstrate that both acquisition as well as long-term retention for AAC in diurnal zebrafish are modulated in a circadian rhythm (Rawashdeh et al., 2007). Animals trained during the subjective day (active phase) showed a significant reduction in the rate of learning compared with animals trained during their inactive phase (subjective night). Furthermore, long-term memory was formed following acquisition during the subjective day, which was not the case for animals acquiring the task during the subjective night. The fact that only animals trained during the subjective day showed good long-term retentions regardless of the time of testing suggested that the induction or formation of long-term memory rather than memory retrieval was modulated by a circadian clock.

Rawashdeh et al. (2007) also demonstrate a possible mechanism by which long-term memory formation in zebrafish varies based on time-of-day; that is via the circadian regulation of endogenous melatonin. This was demonstrated by showing that melatonin treatment of animals in constant darkness during the subjective day (i.e., mimicking subjective night with respect to the presence of elevated melatonin levels), inhibited long-term memory formation. In addition, constant light exposure or pinealectomy, both of which lower endogenous melatonin levels, reversed subjective night inhibition of long-term memory formation. Furthermore, inhibition of melatonin receptor mediated signaling blocked melatonin-induced inhibition of long-term memory formation for acquisition during the subjective day. Finally, blocking endogenous signaling during the subjective night significantly improved long-term memory formation following acquisition for AAC during the subjective night.

The finding that memory can be improved by blocking melatonin signaling during the night for nighttime acquisition encourages further research as a possible drug target for treatment for those patients with abnormal sleep/wake cycles and consequently suffer from deficits in cognitive ability.

\section{Effects of alterations in the circadian system on learning and memory in mutant mice}

In addition to the above mentioned nonmammalian models, there is also growing evidence that the circadian system provides a temporal framework for cognitive function in mammals. This points toward a phylogenetically conserved mechanism, since the ability of a variety of organisms to learn and remember behavioral tasks can fluctuate with the time-of-day (Mistlberger et al., 1996; Valentinuzzi et al., 2000; Chaudhury and Colwell, 2002; Fernandez et al., 2003). Interestingly, the performance of mammals in a variety of learned behavioral tasks is also compromised when sleep is disrupted (Ellenbogen et al., 2006; Wright et al., 2006; Ruby et al., 2008). Recent work has established that the 
genes responsible for driving circadian oscillations are widely expressed in many brain regions, including those involved in learning and memory, such as the amygdala (Lamont et al., 2005), hippocampus (Wakamatsu et al., 2001; Chaudhury et al., 2008; Jilg et al., 2009; Wang et al., 2009), and the ventral tegmental area (McClung et al., 2005; Hampp et al., 2008). Mutations in these circadian clock genes alter performance in a variety of learned behavioral tasks (Garcia et al., 2000; Abarca et al., 2002; Van der Zee et al., 2008; Jilg et al., 2009; Wang et al., 2009). In addition, isolated tissue from the hippocampus continues to exhibit circadian oscillations in culture, and therefore can be considered an endogenous oscillator (Wang et al., 2009). The mechanisms underlying these observations are not well known, but the necessity of the circadian regulation of the PKA/MAPK/cAMP signaling pathway in the hippocampus for memory behavior (Eckel-Mahan et al., 2008) suggests that the involvement of this intracellular transduction cascade within the mammalian nervous system may be similar to what is necessary for the time-ofday regulation of memory in Aplysia (Lyons et al., 2006a).

Work with fear conditioning in mice suggests that memory processes, or the recall of the behavioral task, may be particularly sensitive to disruptions in circadian timing. Deficits in recall of trace, but not cued, fear conditioning, with no effects on acquisition, were observed in period 2 (Per2) gene-mutant mice (Wang et al., 2009). Using this paradigm, conditioned fear response in mice deficient in the vasoactive intestinal peptide (VIP) was also analyzed. VIP is a neuropeptide that is critical to cellular communication within the circadian system and the loss of this peptide results in an extremely compromised circadian system (Colwell et al., 2003; Aton et al., 2005; Ciarleglio et al., 2009). Chaudhury et al. (2008) found that while VIP-deficient mice showed comparable acquisition and recall at $24 \mathrm{~h}$ to controls, VIP-mutants exhibited significant reduction in recall when tested $48 \mathrm{~h}$ or longer after training. Surprisingly, VIP-deficient mice continued to express circadian rhythms in the recall of the training even in those individual mice whose wheel running wheel activity was arrhythmic. One mechanistic explanation is suggested by the finding that daily rhythms in the expression of the Per2 gene continue in the hippocampus of VIP-deficient mice. This suggests that different behaviors could be temporally regulated by circadian oscillators outside of the master pacemaker, the suprachiasmatic nucleus (SCN). Previous work has established that an intact SCN is not required for at least some circadian rhythms in learned behaviors (Mistlberger et al., 1996; Cain and Ralph, 2009). This raises the possibility that circadian pacemakers outside of the SCN may directly drive the rhythms in learned behavior, and that coordination between different circadian pacemakers within the nervous system may be critical for optimal performance. One method to investigate this possibility would be by transiently disrupting the circadian system through rapid changes in the light-dark cycle, similar to the protocol used previously to cause transient desynchrony between the SCN and peripheral oscillators (Davidson et al., 2009). New work presented by Loh et al. (2009) at the Society for Neuroscience meeting, using this model, show that recall of the training is selectively disrupted (see also Craig and McDonald, 2008). These data suggest that past experience with rapid shifts in the LD cycle helps counter the effects of phase disruption on fear conditioning. Therefore one important outcome of this work may be to develop alternative strategies to preserve cognitive abilities following disruptions in sleep and circadian rhythms.

\section{Activation of the MAPK pathway oscillates in a circadian manner in the hippocampus}

While once thought to keep time only in restricted brain regions, it is clear that circadian clocks are more ubiquitous in the CNS. Many studies have addressed the effects of circadian time on long-term memory (Devan et al., 2001; Chaudhury and Colwell, 2002; Lyons et al., 2006b), but the molecular explanations for its contribution have been difficult to identify. The hippocampus, a region of the brain important for the acquisition, consolidation, and temporary storage of long-term memory, hosts $24 \mathrm{~h}$ oscillations in activation of the MAPK pathway (Eckel-Mahan et al., 2008). Consistent with other endogenous circadian rhythms, oscillations in MAPK activation persist in the hippocampus in freerunning (constant dark) conditions. Activation of the MAPK pathway, which is stimulated by the calcium-sensitive cyclases in hippocampal neurons (Sindreu et al., 2007), is a pathway that is required during both the consolidation and reconsolidation phases of some forms of hippocampus-dependent memory (Atkins et al., 1998; Kelly et al., 2003); therefore, its activity variance may be critical for behavior. Its oscillatory nature, not merely the presence of activation in the hippocampus, appears to be important for the persistence of fear memory, indicating that it may be one of the mechanisms by which the circadian clock impinges on fear memory. The contribution of circadian MAPK activation to memory persistence is interesting, considering the role that sleep is thought to play in memory reactivation and its subsequent consolidation (Marshall and Born, 2007; Stickgold and Walker, 2007). It is certainly possible that cycling MAPK activation in the hippocampus is required for its engagement during sleep and that blocking its activity peak interferes with sleep-dependent memory processing. Increasing MAPK activity at its circadian nadir has a similar effect on memory persistence, however, underscoring the importance of circadian cycling of this pathway on memory-specific ensembles.

Whether oscillations in cAMP and MAPK activity in the hippocampus contribute to core clock gene expression in the hippocampus is not known. Observations of robust clock gene expression in the hippocampus do exist, but how they contribute to synaptic plasticity in the hippocampus is still unclear (Garcia et al., 2000; Wakamatsu et al., 2001; Jilg et al., 2009). Activation of the MAPK pathway oscillates in the circadian pacemaker (SCN), is light responsive, and can phase-shift subsequent circadian clock rhythms in SCN neurons as well as in other CNS and peripheral tissues (Obrietan et al., 1998; Butcher et al., 2002). The circadian oscillation of MAPK activation in the hippocampus and its memory-responsiveness raise questions as to whether training might function similarly in hippocampal neurons as light activation and clock resetting functions in SCN neurons; inducing a unique, temporally specific molecular profile in memory-specific neuronal ensembles.

\section{Sleep and circadian regulation of memory and cognitive performance}

Human cognition is modulated by interactions between sleepwake homeostasis, sleep inertia, and the internal circadian clock. The homeostatic drive for sleep builds up with time awake (Czeisler et al., 1994; Borbély and Achermann, 1999); sleep inertia is the impairment in performance observed immediately upon awakening (Balkin et al., 2002; Wertz et al., 2006), and the circadian clock drives near $24 \mathrm{~h}$ rhythms in performance (Czeisler et al., 1994; Wyatt et al., 1999). These fundamental CNS processes interact in a nonlinear manner to modulate human physiology and behavior. Under normal entrained conditions, performance 
improves across the first few hours awake as sleep inertia dissipates and the circadian system promotes wakefulness in the latter half of the day to counter the buildup of homeostatic sleep drive. After sleep inertia is dissipated, the net result of the interaction between homeostatic sleep drive and the circadian clock is to maintain relatively stable levels of human performance for 15-17 h per day. Extended wakefulness leads to impaired performance (Durmer and Dinges, 2005).

Czeisler et al. (1994) and Wright et al. (2002) modeled the individual contribution of these components using a forced desynchrony protocol (Czeisler et al., 1999; Wright et al., 2001). The forced desynchrony is an experimental protocol that schedules subjects to live on day lengths beyond the range of entrainment to which the internal circadian clock can adapt. This protocol permits examination of the interaction between time awake and circadian influences on human cognition as scheduled wakefulness and sleep occur across the circadian cycle. Findings from such studies demonstrate that the difference between the best and worst performance across the circadian cycle is greatest when homeostatic sleep drive is high and is smallest when homeostatic sleep drive is low. Furthermore, performance is low in humans during the biological night when endogenous levels of the clockcontrolled sleep-promoting hormone melatonin are high. Additionally, sleep inertia can impair performance as much as sleep deprivation. Stable individual differences in cognitive impairment due to sleepiness are observed and these individual differences appear to be task dependent.

Disruption of the phase relationship between sleep inertia, sleep homeostatic, and circadian processes leads to impairments in performance that negatively impact work/school productivity and public safety (e.g., drowsy driving and flying). In fact, failure to adapt the internal circadian clock to work schedules associated with altered work environments (e.g., schedules kept by medical residents and other shift workers, airline pilots, astronauts, polar expedition, security, and military personnel) leads to impaired learning and performance (Dijk et al., 2001; Lockley et al., 2004; Lamond et al., 2006; Wright et al., 2006). Studies aimed at characterizing the molecular players involved in mediating sleepwakefulness and circadian processes modulating cognition, such as melatonin and clock genes, are important for understanding physiological mechanisms underlying patterns of high and low performance, as well as for the development of effective countermeasures to combat sleepiness.

\section{Conclusions}

The mini-symposium presentations summarized above provide examples of how endogenous circadian rhythms can strongly modulate cognitive processes. This modulation exists across phylogeny, for different types of memories, and in different memory processes. An understanding of the molecular mechanisms that regulate the circadian expression of memory formation are only beginning to be understood, but seem to include melatonin, the MAPK cascade, and clock genes such as period. Furthermore, it seems that the proper oscillation of the MAPK cascade seems to be necessary for the persistence of memory, suggesting that this pathway is not only involved in the time-of-day expression of memory, but also that the cycling of MAPK activity itself is needed for the proper consolidation of the memory. Events downstream of the MAPK cascade, such as activation of the cAMP-responsive element binding protein (CREB) transcriptional pathway, appear to be also gated by the circadian clock (Belvin et al., 1999), and new data presented at the 2009 annual meeting of the Society for Neuroscience suggest specific oscilla- tion times may permit CREB-mediated enhancement of memory (Gonzales et al., 2009; Yin et al., 2009). Study of the mechanistic relationship between melatonin, and the MAPK/CREB cascade, with clock genes such as period are needed for our basic understanding of the underlying functional events that regulate memory formation and circadian rhythms. This mini-symposium highlights the importance of the use of various animal models to study the time-of-day regulation of memory. The comparative molecular neurobiology underscores evolutionarily conserved (and therefore significant) processes for circadian memory modulation. Indeed, studies have already begun which describe functional interactions between melatonin cycling and period 3 polymorphisms in human cognitive performance and sleep (Groeger et al., 2008). In our ever increasing $24 \mathrm{~h}$ society, disruption of normal circadian rhythms, such as a result from irregular shift-work, seems to have negative consequences in cognitive function, and therefore has negative implications for public safety, health, and overall work performance output. Future studies examining the role of circadian rhythm biology on memory and cognitive performance will aid our understanding of the implications that our $24 \mathrm{~h}$ society presents, and will undoubtedly help guide treatments for various cognitive and sleep-related disorders.

\section{References}

Abarca C, Albrecht U, Spanagel R (2002) Cocaine sensitization and reward are under the influence of circadian genes and rhythm. Proc Natl Acad Sci U S A 99:9026-9030.

Andretic R, Chaney S, Hirsh J (1999) Requirement of circadian genes for cocaine sensitization in Drosophila. Science 285:1066-1068.

Antoniadis EA, Ko CH, Ralph MR, McDonald RJ (2000) Circadian rhythms, aging and memory. Behav Brain Res 114:221-233.

Atkins CM, Selcher JC, Petraitis JJ, Trzaskos JM, Sweatt JD (1998) The MAPK cascade is required for mammalian associative learning. Nat Neurosci 1:602-609.

Aton SJ, Colwell CS, Harmar AJ, Waschek J, Herzog ED (2005) Vasoactive intestinal polypeptide mediates circadian rhythmicity and synchrony in mammalian clock neurons. Nat Neurosci 8:476-483.

Balkin TJ, Braun AR, Wesensten NJ, Jeffries K, Varga M, Baldwin P, Belenky G, Herscovitch P (2002) The process of awakening: A PET study of regional brain activity patterns mediating the re-establishment of alertness and consciousness. Brain 125:2308-2319.

Bargiello TA, Jackson FR, Young MW (1984) Restoration of circadian behavioural rhythms by gene transfer in Drosophila. Nature 312:752-754.

Barnes CA, McNaughton BL, Goddard GV, Douglas RM, Adamec R (1977) Circadian rhythm of synaptic excitability in rat and monkey central nervous system. Science 197:91-92.

Beck CD, Schroeder B, Davis RL (2000) Learning performance of normal and mutant Drosophila after repeated conditioning trials with discrete stimuli. J Neurosci 20:2944-2953.

Belvin MP, Zhou H, Yin JC (1999) The drosophila dCREB2 gene affects the circadian clock. Neuron 22:777-787.

Borbély AA, Achermann P (1999) Sleep homeostasis and models of sleep regulation. J Biol Rhythms 14:557-568.

Butcher GQ, Doner J, Dziema H, Collamore M, Burgoon PW, Obrietan K (2002) The p42/44 mitogen-activated protein kinase pathway couples photic input to circadian clock entrainment. J Biol Chem 277:29519-29525.

Cahill GM, Hurd MW, Batchelor MM (1998) Circadian rhythmicity in the locomotor activity of larval zebrafish. Neuroreport 9:3445-3449.

Cain SW, Ralph MR (2009) Circadian modulation of conditioned place avoidance in hamsters does not require the suprachiasmatic nucleus. Neurobiol Learn Mem 91:81-84.

Castillo MR, Hochstetler KJ, Greene DM, Firmin SI, Tavernier RJ, Raap DK, Bult-Ito A (2005) Circadian rhythm of core body temperature in two laboratory mouse lines. Physiol Behav 86:538-545.

Chaudhury D, Colwell CS (2002) Circadian modulation of learning and memory in fear-conditioned mice. Behav Brain Res 133:95-108.

Chaudhury D, Loh DH, Dragich JM, Hagopian A, Colwell CS (2008) Select 
cognitive deficits in vasoactive intestinal peptide deficient mice. BMC Neurosci 9:63.

Ciarleglio CM, Gamble KL, Axley JC, Strauss BR, Cohen JY, Colwell CS, McMahon DG (2009) Population encoding by circadian clock neurons organizes circadian behavior. J Neurosci 29:1670-1676.

Colwell CS, Michel S, Itri J, Rodriguez W, Tam J, Lelievre V, Hu Z, Liu X, Waschek JA (2003) Disrupted circadian rhythms in VIP- and PHIdeficient mice. Am J Physiol Regul Integr Comp Physiol 285:R939-R949.

Costa-Mattioli M, Sossin WS, Klann E, Sonenberg N (2009) Translational control of long-lasting synaptic plasticity and memory. Neuron 61:10-26.

Craig LA, McDonald RJ (2008) Chronic disruption of circadian rhythms impairs hippocampal memory in the rat. Brain Res Bull 76:141-151.

Czeisler CA, Dijk DJ, Duffy JF (1994) Entrained phase of the circadian pacemaker serves to stabilize alertness and performance throughout the habitual waking day. In: Sleep onset: normal and abnormal processes (Ogilvie RD, Harsh JR eds), pp 89-110. Washington, D.C.: American Psychological Association.

Czeisler CA, Duffy JF, Shanahan TL, Brown EN, Mitchell JF, Rimmer DW, Ronda JM, Silva EJ, Allan JS, Emens JS, Dijk DJ, Kronauer RE (1999) Stability, precision, and near-24-hour period of the human circadian pacemaker. Science 284:2177-2181.

Davidson AJ, Castanon-Cervantes O, Leise TL, Molyneux PC, Harrington ME (2009) Visualizing jet lag in the mouse suprachiasmatic nucleus and peripheral circadian timing system. Eur J Neurosci 29:171-180.

Decker S, McConnaughey S, Page TL (2007) Circadian regulation of insect olfactory learning. Proc Natl Acad Sci U S A 104:15905-15910.

Devan BD, Goad EH, Petri HL, Antoniadis EA, Hong NS, Ko CH, Leblanc L, Lebovic SS, Lo Q, Ralph MR, McDonald RJ (2001) Circadian phaseshifted rats show normal acquisition but impaired long-term retention of place information in the water task. Neurobiol Learn Mem 75:51-62.

Dijk DJ, Neri DF, Wyatt JK, Ronda JM, Riel E, Ritz-De Cecco A, Hughes RJ, Elliott AR, Prisk GK, West JB, Czeisler CA (2001) Sleep, performance, circadian rhythms, and light-dark cycles during two space shuttle flights. Am J Physiol Regul Integr Comp Physiol 281:R1647-R1664.

Durmer JS, Dinges DF (2005) Neurocognitive consequences of sleep deprivation. Semin Neurol 25:117-129.

Eckel-Mahan KL, Phan T, Han S, Wang H, Chan GC, Scheiner ZS, Storm DR (2008) Circadian oscillation of hippocampal MAPK activity and cAmp: Implications for memory persistence. Nat Neurosci 11:1074-1082.

Ellenbogen JM, Payne JD, Stickgold R (2006) The role of sleep in declarative memory consolidation: Passive, permissive, active or none? Curr Opin Neurobiol 16:716-722.

Fekete M, van Ree JM, Niesink RJ, de Wied D (1985) Disrupting circadian rhythms in rats induces retrograde amnesia. Physiol Behav 34:883-887.

Fernandez RI, Lyons LC, Levenson J, Khabour O, Eskin A (2003) Circadian modulation of long-term sensitization in Aplysia. Proc Natl Acad Sci U S A 100:14415-14420.

Gallego M, Virshup DM (2007) Post-translational modifications regulate the ticking of the circadian clock. Nat Rev Mol Cell Biol 8:139-148.

Garcia JA, Zhang D, Estill SJ, Michnoff C, Rutter J, Reick M, Scott K, DiazArrastia R, McKnight SL (2000) Impaired cued and contextual memory in NPAS2-deficient mice. Science 288:2226-2230.

Gerard RW (1949) Physiology and psychiatry. Am J Psychiatry 106:161-173.

Gonzales ED, Tubon TC, Drier EA, Jin H, Gerstner JR, Zhou H, Drier D, Rutecki PA, Yin JC (2009) dCREB2-mediated enhancement of memory formation. Soc Neurosc Abstr 35:497.7.

Green C, Michel M, Lyons LC (2009) Differential regulation of intermediate and long-term associative memory by the circadian clock. Soc Neurosc Abstr 35:888.14/GG20.

Groeger JA, Viola AU, Lo JC, von Schantz M, Archer SN, Dijk DJ (2008) Early morning executive functioning during sleep deprivation is compromised by a PERIOD3 polymorphism. Sleep 31:1159-1167.

Hampp G, Ripperger JA, Houben T, Schmutz I, Blex C, Perreau-Lenz S, Brunk I, Spanagel R, Ahnert-Hilger G, Meijer JH, Albrecht U (2008) Regulation of monoamine oxidase A by circadian-clock components implies clock influence on mood. Curr Biol 18:678-683.

Hebb DO (1949) The organization of behavior; a neuropsychological theory. New York, Wiley.

Hurd MW, Debruyne J, Straume M, Cahill GM (1998) Circadian rhythms of locomotor activity in zebrafish. Physiol Behav 65:465-472.

Jilg A, Lesny S, Peruzki N, Schwegler H, Selbach O, Dehghani F, Stehle JH
(2009) Temporal dynamics of mouse hippocampal clock gene expression support memory processing. Hippocampus, in press.

Johnson JH, Clemens JA, Terkel J, Whitmoyer DI, Sawyer CH (1972) Technique for recording multiple-unit activity from the brain of the freelymoving rat. Neuroendocrinology 9:90-99.

Kelly A, Laroche S, Davis S (2003) Activation of mitogen-activated protein kinase/extracellular signal-regulated kinase in hippocampal circuitry is required for consolidation and reconsolidation of recognition memory. J Neurosci 23:5354-5360.

Konopka RJ, Benzer S (1971) Clock mutants of Drosophila melanogaster. Proc Natl Acad Sci U S A 68:2112-2116.

Konopka RJ, Pittendrigh C, Orr D (1989) Reciprocal behaviour associated with altered homeostasis and photosensitivity of Drosophila clock mutants. J Neurogenet 6:1-10.

Krishnan B, Levine JD, Lynch MK, Dowse HB, Funes P, Hall JC, Hardin PE, Dryer SE (2001) A new role for cryptochrome in a Drosophila circadian oscillator. Nature 411:313-317.

Lamond N, Petrilli RM, Dawson D, Roach GD (2006) Do short international layovers allow sufficient opportunity for pilots to recover? Chronobiol Int 23:1285-1294.

Lamont EW, Robinson B, Stewart J, Amir S (2005) The central and basolateral nuclei of the amygdala exhibit opposite diurnal rhythms of expression of the clock protein Period2. Proc Natl Acad Sci U S A 102: $4180-4184$.

Lockley SW, Cronin JW, Evans EE, Cade BE, Lee CJ, Landrigan CP, Rothschild JM, Katz JT, Lilly CM, Stone PH, Aeschbach D, Czeisler CA, Harvard Work Hours, Health and Safety Group (2004) Effect of reducing interns' weekly work hours on sleep and attentional failures. N Engl J Med 351:1829-1837.

Loh DH, Wang LM, Chaudhury D, Colwell CS (2009) Effects of alterations in the circadian system on learning and memory. Soc Neurosci Abstr 35:797.6.

Lyons LC, Roman G (2009) Circadian modulation of short-term memory in Drosophila. Learn Mem 16:19-27.

Lyons LC, Rawashdeh O, Katzoff A, Susswein AJ, Eskin A (2005) Circadian modulation of complex learning in diurnal and nocturnal Aplysia. Proc Natl Acad Sci U S A 102:12589-12594.

Lyons LC, Collado MS, Khabour O, Green CL, Eskin A (2006a) The circadian clock modulates core steps in long-term memory formation in Aplysia. J Neurosci 26:8662-8671.

Lyons LC, Rawashdeh O, Eskin A (2006b) Non-ocular circadian oscillators and photoreceptors modulate long term memory formation in Aplysia. J Biol Rhythms 21:245-255.

Lyons LC, Green CL, Eskin A (2008) Intermediate-term memory is modulated by the circadian clock. J Biol Rhythms 23:538-542.

Marrus SB, Zeng H, Rosbash M (1996) Effect of constant light and circadian entrainment of perS flies: Evidence for light-mediated delay of the negative feedback loop in Drosophila. EMBO J 15:6877-6886.

Marshall L, Born J (2007) The contribution of sleep to hippocampusdependent memory consolidation. Trends Cogn Sci 11:442-450.

McClung CA, Sidiropoulou K, Vitaterna M, Takahashi JS, White FJ, Cooper DC, Nestler EJ (2005) Regulation of dopaminergic transmission and cocaine reward by the clock gene. Proc Natl Acad Sci U S A 102:9377-9381.

McGaugh JL (2000) Memory-a century of consolidation. Science 287:248-251.

Mistlberger RE, de Groot MH, Bossert JM, Marchant EG (1996) Discrimination of circadian phase in intact and suprachiasmatic nuclei-ablated rats. Brain Res 739:12-18.

Monk TH, Folkard S (1978) Concealed inefficiency of late-night study. Nature 273:296-297.

Obrietan K, Impey S, Storm DR (1998) Light and circadian rhythmicity regulate MAP kinase activation in the suprachiasmatic nuclei. Nat Neurosci 1:693-700.

Panda S, Hogenesch JB, Kay SA (2002) Circadian rhythms from flies to human. Nature 417:329-335.

Rawashdeh O, de Borsetti NH, Roman G, Cahill GM (2007) Melatonin suppresses nighttime memory formation in zebrafish. Science 318:11441146.

Reddy P, Zehring WA, Wheeler DA, Pirrotta V, Hadfield C, Hall JC, Rosbash M (1984) Molecular analysis of the period locus in Drosophila melanogaster and identification of a transcript involved in biological rhythms. Cell 38:701-710. 
Roman G, Davis RL (2001) Molecular biology and anatomy of Drosophila olfactory associative learning. Bioessays 23:571-581.

Roth TL, Sweatt JD (2008) Rhythms of memory. Nat Neurosci 11:993-994.

Ruby NF, Hwang CE, Wessells C, Fernandez F, Zhang P, Sapolsky R, Heller HC (2008) Hippocampal-dependent learning requires a functional circadian system. Proc Natl Acad Sci U S A 105:15593-15598.

Rudy JW, Pugh CR (1998) Time of conditioning selectively influences contextual fear conditioning: Further support for a multiple-memory systems view of fear conditioning. J Exp Psychol Anim Behav Process 24:316-324.

Sakai T, Tamura T, Kitamoto T, Kidokoro Y (2004) A clock gene, period, plays a key role in long-term memory formation in Drosophila. Proc Natl Acad Sci U S A 101:16058-16063.

Scheving LE, Harrison WH, Gordon P, Pauly JE (1968) Daily fluctuation (circadian and ultradian) in biogenic amines of the rat brain. Am J Physiol 214:166-173.

Sindreu CB, Scheiner ZS, Storm DR (2007) $\mathrm{Ca}^{2+}$-stimulated adenylyl cyclases regulate ERK-dependent activation of MSK1 during fear conditioning. Neuron 53:79-89.

Stickgold R, Walker MP (2007) Sleep-dependent memory consolidation and reconsolidation. Sleep Med 8:331-343.

Stokkan KA, Yamazaki S, Tei H, Sakaki Y, Menaker M (2001) Entrainment of the circadian clock in the liver by feeding. Science 291:490-493.

Tapp WN, Holloway FA (1981) Phase shifting circadian rhythms produces retrograde amnesia. Science 211:1056-1058.

Tully T, Quinn WG (1985) Classical conditioning and retention in normal and mutant Drosophila melanogaster. J Comp Physiol A 157:263-277.

Valentinuzzi VS, Buxton OM, Chang AM, Scarbrough K, Ferrari EA, Takahashi JS, Turek FW (2000) Locomotor response to an open field during C57BL/6J active and inactive phases: Differences dependent on conditions of illumination. Physiol Behav 69:269-275.

Valentinuzzi VS, Menna-Barreto L, Xavier GF (2004) Effect of circadian phase on performance of rats in the morris water maze task. J Biol Rhythms 19:312-324.

Van der Zee EA, Havekes R, Barf RP, Hut RA, Nijholt IM, Jacobs EH, Gerkema MP (2008) Circadian time-place learning in mice depends on cry genes. Curr Biol 18:844-848.

Wakamatsu H, Yoshinobu Y, Aida R, Moriya T, Akiyama M, Shibata S (2001) Restricted-feeding-induced anticipatory activity rhythm is associated with a phase-shift of the expression of mPer1 and mPer2 mRNA in the cerebral cortex and hippocampus but not in the suprachiasmatic nucleus of mice. Eur J Neurosci 13:1190-1196.

Wang LM, Dragich JM, Kudo T, Odom IH, Welsh DK, O’Dell TJ, Colwell CS (2009) Expression of the circadian clock gene Period2 in the hippocampus: Possible implications for synaptic plasticity and learned behaviour. ASN Neuro. Advance online publication. Retrieved June 10, 2009. doi: 10.1042/AN20090020.

Wertz AT, Ronda JM, Czeisler CA, Wright KP Jr (2006) Effects of sleep inertia on cognition. JAMA 295:163-164.

Wright KP Jr, Hughes RJ, Kronauer RE, Dijk DJ, Czeisler CA (2001) Intrinsic near-24-h pacemaker period determines limits of circadian entrainment to a weak synchronizer in humans. Proc Natl Acad Sci U S A 98:14027-14032.

Wright KP Jr, Hull JT, Czeisler CA (2002) Relationship between alertness, performance, and body temperature in humans. Am J Physiol Regul Integr Comp Physiol 283:R1370-R1377.

Wright KP Jr, Hull JT, Hughes RJ, Ronda JM, Czeisler CA (2006) Sleep and wakefulness out of phase with internal biological time impairs learning in humans. J Cogn Neurosci 18:508-521.

Wyatt JK, Ritz-De Cecco A, Czeisler CA, Dijk DJ (1999) Circadian temperature and melatonin rhythms, sleep, and neurobehavioral function in humans living on a 20-h day. Am J Physiol 277:R1152-R1163.

Yin JC, Gerstner JR, Elliott JL, Drier EA, Swanson JC, Gonzales ED, Zhou H, Tubon TC (2009) Dynamic oscillations in dCREB2 activity across the day/night cycle. Soc Neurosci Abstr 35:497.6. 